\title{
Identificación de territorios críticos en salud materna mediante indicadores
}

\author{
Marcos Paz Ballivián ${ }^{1}$ y Alberto de la Gálvez Murillo-Camberos ${ }^{1}$
}

RESUMEN Objetivos. El Ministerio de Salud y Previsión Social de Bolivia convocó a principios de 2000 a varios expertos para que, a partir de la información disponible, elaboraran un método que permitiera identificar territorios críticos en un tema de por sí prioritario - la salud materna y neonatal-y crear un mapa de la situación y de la capacidad instalada en las 112 provincias nacionales. En este estudio se describe el método utilizado.

Métodos. Se crearon indices de la situación de salud y de la capacidad instalada. Los pasos seguidos en este proceso fueron los siguientes: 1) identificación de las variables incluidas en cada índice; 2) ponderación de las variables de cada índice; creación de una fórmula para cada indice; 4) elaboración de una planilla con los datos de cada provincia correspondientes a las variables elegidas y al valor porcentual provincial de cada indice, obtenido mediante la aplicación de la respectiva fórmula; 5) identificación de tres categorías continuas para cada índice, y 6) definición de la taxonomía.

Resultados. De este modo, se obtuvo un mapa nacional de la situación de salud materna y de la capacidad instalada en cada una de las 112 provincias bolivianas, lo cual permitió seleccionar un número mínimo de provincias para realizar intervenciones conjuntas entre el Ministerio de Salud y Previsión Social y otras instituciones. Las 9 provincias seleccionadas tienen un total de 26 municipios que implican a 17 distritos sanitarios y concentran el $29 \%$ de la población del país, el 33\% de las muertes maternas y el 35\% de las defunciones neonatales tempranas estimadas.

Conclusiones. Basándose en información disponible, el método utilizado proporcionó un mapa de la situación global, en este caso de la salud materna, de las 112 provincias de los 9 departamentos bolivianos que permite identificar territorios críticos para las intervenciones en materia de salud.

Palabras clave Salud materna, salud neonatal, capacidad instalada, índices de la situación de salud.

Las reformas del Estado, y en particular la descentralización, han modificado, o lo están haciendo, las reglas de juego y el papel de los actores institu-

\footnotetext{
1 Proyecto Salud Materna y Neonatal, Programa Internacional de Educación en Ginecología y Obstetricia de la Universidad Johns Hopkins (JHPIEGO), La Paz, Bolivia. La correspondencia debe ser enviada a Marcos Paz a la siguiente dirección: Calle Ricardo Mujía N ${ }^{\circ} 754, \mathrm{La} \mathrm{Paz}$, Bolivia. Correo electrónico: mpaz@jhpiego.org.bo
}

cionales y sociales involucrados en la gestión de los recursos de salud. Así, los niveles centrales de los ministerios de salud, antes concentradores y en gran medida ejecutores directos, han visto la necesidad de reforzar su papel normativo y de evaluación, y de establecer unas pocas estrategias de salud globales en la línea de favorecer la equidad en materia de salud. Paralelamente al establecimiento de líneas estratégicas de acción a partir de la de- finición de prioridades nacionales, habitualmente inscritas en el marco de las iniciativas internacionales globales (maternidad segura, por ejemplo), los niveles centrales de los ministerios de salud, por su capacidad de generar apoyo financiero externo, tienen la posibilidad adicional de concentrar esfuerzos en territorios más concretos, a condición de catalogarlos como prioritarios, por el impacto esperado de las intervenciones. 
Sin embargo, la identificación de esos territorios puede responder a factores subjetivos, a intereses o a facilidades geográficas de acceso, determinando un efecto poco relevante de las acciones emprendidas. Por lo tanto, es una necesidad, y al mismo tiempo un desafío, establecer un procedimiento o método para identificar territorios críticos para acciones globales o específicas para ciertos problemas de salud, tanto para comprender cualitativa y cuantitativamente la dimensión del problema, como para medir el progreso de los programas y servicios y establecer en qué medida están respondiendo a las necesidades de salud de la población $(1,2)$. El desafío pasa también por el reconocimiento de la disponibilidad limitada de información y por la poca factibilidad de establecer procesos de recolección y procesamiento de datos muy precisos y con los niveles de desagregación requeridos por el sector. Además, supone una interpretación previa de las posibilidades de generar con éxito voluntades políticas y esfuerzos de coordinación y regulación de dichas voluntades.

Bolivia está dividida en nueve departamentos, que a su vez se subdividen en 112 provincias. Cada provincia está formada por municipios, que son la unidad territorial y administrativa a cargo del gobierno municipal, elegido por voto ciudadano cada cinco años. Hay 314 municipios. Los distritos de salud son una división particular del sector, que en general no respeta la división política.

En Bolivia, la salud materna y neonatal sigue siendo un tema de preocupación general, en especial por las elevadas tasas de defunción. En lo que se refiere a la mortalidad materna, la última cifra disponible (390 defunciones por 100000 nacidos vivos), obtenida por el método de supervivencia de hermanas (3), corresponde de manera aproximada al año 1991. Sin embargo, las estimaciones realizadas por la Organización Mundial de la Salud (OMS), el Fondo de las Naciones Unidas para la Infancia (UNICEF) y el Fondo de Población de las Naciones Unidas (FNUAP), mediante ajuste de los datos de la Encuesta Nacional de
Demografía y Salud de 1994 (ENDSA94), asignan a Bolivia una tasa de 550 muertes maternas por 100000 nacidos vivos durante el período 1989-1996, con un recorrido que va de 370 a 740 $(4,5)$. En cuanto a la cobertura del parto en establecimientos de cualquiera de los tres niveles de atención con camas de maternidad (parto en servicios de obstetricia), considerando los sectores público y privado, el país habría cumplido la meta del $60 \%$ de cobertura para el año $2000(5,6)$, acordada en el Plan Regional para la Reducción de la Mortalidad Materna en las Américas $(6,7)$. De acuerdo con la ENDSA-98, en el período 1993-1998 la mortalidad infantil fue de 67 por 1000 nacidos vivos, la mortalidad neonatal global de 34 por 1000 y la mortalidad neonatal precoz de 22 por 1000 (8).

Tal situación dio lugar a una estrategia global de provisión de suministros esenciales para una canasta básica de prestaciones que incluye servicios de salud materna y neonatal, para permitir el acceso gratuito de la población a la atención de salud. La estrategia se conoce como Seguro Básico de Salud (creada en 1995 con el nombre de Seguro de Maternidad y Niñez) y su financiamiento procede de las partidas que el Tesoro General de la Nación asigna anualmente a cada uno de los 314 municipios, mediante un procedimiento de capitación que está respaldado por la Ley de Participación Popular promulgada por el Honorable Congreso Nacional de Bolivia en 1997, la misma que reconoce a las municipalidades funciones de administración e inversión en salud y educación, y que inició el proceso de descentralización de tales sectores. Paralelamente a esta estrategia, el Ministerio de Salud y Previsión Social consideró necesario seleccionar territorios críticos en salud materna y neonatal precoz, con el fin de proporcionarles una intervención más intensiva mediante acciones financiadas por agencias de cooperación, proyectos de salud y organizaciones no gubernamentales (ONG), con el concurso decidido de los gobiernos municipales y las entidades locales. Esto implica que en la práctica, “. . .la descentralización pueda consi- derarse como una manera de identificar mejor las necesidades locales y responder más adecuadamente a ellas, y también como una forma de mejorar la gestión de los recursos" (9).

El procedimiento, inspirado en la filosofía de los Sistemas Locales de Salud y elaborado a partir de datos disponibles, se denomina "Mapeo de Salud Materna y Neonatal" y está permitiendo concentrar esfuerzos de diferentes instituciones en 17 distritos de salud en los que vive el $29 \%$ de la población nacional y se producen el 33\% de las muertes maternas y el $35 \%$ de las defunciones neonatales tempranas estimadas. El procedimiento se basa en la organización y puesta en funcionamiento de redes de servicios y redes sociales con funciones asignadas (10), porque no se puede concebir un sistema local de salud sin una red de servicios adecuada en cantidad y calidad, $\mathrm{y} \sin$ nuevas formas de relacionamiento social que incluyan a todos los actores (2).

A partir de la convocatoria del Ministerio de Salud y Previsión Social, la propuesta inicial, la conducción de las discusiones técnicas y la redacción de los avances y del documento final estuvieron a cargo del Proyecto Salud Materna y Neonatal del Programa Internacional de Educación en Ginecología y Obstetricia de la Universidad Johns Hopkins de Baltimore, Estados Unidos (Johns Hopkins Program of International Education in Gynecology and Obstetrics: JHPIEGO). El "Mapeo de Salud Materna y Neonatal" fue una iniciativa concertada con el Componente de Intervenciones Estratégicas Localizadas (CIELO) del Proyecto de Salud Integral (PROSIN), financiado por la Agencia para el Desarrollo Internacional de Estados Unidos (USAID). El FNUAP, con su Programa de Salud Sexual y Reproductiva, ha convenido en apoyar la realización de acciones en estos distritos, del mismo modo que las cooperaciones canadiense e italiana. Las ONG de la red PROCOSI (Proyecto de Cooperación en Salud Integral) y otras también han decidido asociarse con este movimiento. La Fundación Bill y Melinda Gates, mediante Save the Children, ha preparado un proyecto de 
salud con énfasis en la atención del neonato a base del mapeo.

Este artículo tiene el propósito de difundir el método utilizado en el "mapeo de la salud materna y neonatal", referido únicamente al componente materno, porque puede ayudar a definir prioridades en salud y a forjar alianzas.

\section{MATERIALES Y MÉTODOS}

En primer lugar se identificaron las variables necesarias para el mapeo de salud materna por territorio provincial. Es decir, datos de producción de servicios, procedentes del Sistema Nacional de Información en Salud (11); proyecciones de población para 1999, del Instituto Nacional de Estadística (12); Índices de Desarrollo Humano por municipio, elaborados por el Ministerio de Desarrollo Sostenible y el PNUD a partir de la esperanza de vida al nacer; tasa de alfabetización; años de escolaridad; producto interno bruto per cápita (13), y tasas de mortalidad materna (ENDSA-94) (3) y neonatal (ENDSA-98) (8).

A continuación se eligieron cuatro variables para crear un índice de la situación de la salud materna (ISSM) y otras cuatro para crear un índice de la capacidad instalada (ICI). Las variables del ISSM son de registro periódico y también son las recomendadas como indicadores de proceso en el análisis de la mortalidad materna y la iniciativa de maternidad segura $(1,14,15)$. A continuación se procedió a la ponderación de cada variable de ambos índices sobre un total de 100 puntos (cuadro 1). Dicha ponderación, a pesar de ser arbitraria, fue fruto del consenso entre los expertos. La ponderación, además de que implica el peso asignado a cada variable, permite desarrollar una fórmula, que es el paso siguiente para calcular el índice respectivo.

Considerando la persistencia de un gran grupo de población que sigue con la práctica del parto domiciliario, generalmente sin posibilidades de reaccionar oportunamente a las emergencias obstétricas y neonatales, y siendo el domicilio el lugar más frecuente de

CUADRO 1. Variables de los índices de la situación de salud materna (ISSM) y de la capacidad instalada (ICl) y su respectiva ponderación

\begin{tabular}{|c|c|c|c|}
\hline \multicolumn{2}{|l|}{ ISSM } & \multicolumn{2}{|l|}{$\mathrm{ICl}$} \\
\hline Variables & Ponderación & Variables & Ponderación \\
\hline $\begin{array}{l}\text { Tasa de mortalidad } \\
\text { materna }\end{array}$ & 15 & $\begin{array}{l}\text { Porcentaje de ocupación } \\
\text { de camas de maternidad }\end{array}$ & 20 \\
\hline Número de muertes & & Número de médicos por & \\
\hline maternas & 30 & 10000 habitantes & 20 \\
\hline Índice de desarrollo humano & 25 & Número de hospitales por & \\
\hline Porcentaje de partos & & 10000 habitantes & 30 \\
\hline en servicios de obstetricia & 30 & Porcentaje de partos por cesárea & 30 \\
\hline
\end{tabular}

muerte materna, se le otorgó gran peso al porcentaje de partos en servicios de obstetricia. El número de muertes maternas también mereció una puntuación alta por la necesidad de identificar espacios territoriales de alta mortalidad materna. El número de muertes maternas en cada provincia se calculó aplicando la tasa de mortalidad al número de recién nacidos vivos esperado para el año 1999. También se incluyó un resumen de las capacidades sociales para enfrentar la pobreza, reflejado en el índice de desarrollo humano. La ENDSA-94 calculó ocho tasas de mortalidad materna: nacional, urbana, rural, altiplano, valles, llanos, altiplano urbano y altiplano rural (3). Como esas tasas eran las únicas disponibles, y aun a sabiendas de que pudo haber ocurrido una modificación de las mismas, se asignó alguna de ellas a cada provincia, en función de la concentración demográfica y de su ubicación agroecológica. Para algunas capitales de departamento se utilizaron las tasas aportadas por investigaciones puntuales (16). Las tasas de mortalidad materna y el número de muertes maternas no constituyen una duplicación, sino que responden a la necesidad de controlar de alguna manera las fluctuaciones de la tasa (14) por efecto de la variación, especialmente del numerador. Para la tasa de mortalidad materna y el número de muertes maternas, se otorgó la máxima puntuación (15 y 30 puntos, respectivamente) a las provincias con las cifras más elevadas. En cambio, para el índice de desarrollo humano y la cobertura de par- tos en servicios de obstetricia, fueron las provincias con la cifras más bajas las que recibieron la máxima puntuación (25 y 30 puntos, respectivamente).

En lo que respecta al ICI, las mayores puntuaciones de las cuatro variables correspondieron a las provincias con las cifras más elevadas. Las ponderaciones se asignaron considerando los datos de subutilización de camas de maternidad en muchas regiones, la concentración de la formación profesional en la atención de emergencias en el plantel médico del país, la existencia de hospitales con funciones obstétricas y neonatales esenciales $y$, finalmente, la capacidad real de intervención quirúrgica para resolver las complicaciones más frecuentes. Estas últimas variables tienen mayor peso para medir la capacidad instalada por la eficacia de las intervenciones. Para la primera variable se consideró el número de días-cama de maternidad disponibles en los tres niveles de atención. El número de médicos se expresó por 10000 y no por 1000 habitantes, a fin de obtener cifras iguales a o mayores de uno. Para la variable hospitales, se consideraron los especializados en maternidad (6 institutos), los hospitales generales con servicios de maternidad (40) y los hospitales de distrito (147). Para el porcentaje de cesáreas, que en realidad no valora el exceso o defecto del procedimiento, sino la existencia de capacidad institucional para realizarlas, las provincias con un porcentaje de cesáreas superior al 10\% recibieron 30 puntos; 20 puntos aquellas con un porcentaje entre el $5 \mathrm{y}$ el 
CUADRO 2. Categorización de los índices de la situación de salud materna (ISSM) y de la capacidad instalada (ICI) según la puntuación alcanzada por la suma de las variables que los componen

\begin{tabular}{lrr}
\hline Categoría & $\begin{array}{c}\text { ISSM } \\
\text { (puntos) }\end{array}$ & \multicolumn{1}{c}{$\begin{array}{c}\text { ICl } \\
\text { (puntos) }\end{array}$} \\
\hline Buena & $<25$ & $41-100$ \\
Regular & $25-45$ & $20-40$ \\
Mala & $46-100$ & $<20$ \\
\hline
\end{tabular}

$10 \%$, y 10 puntos aquellas con menos de un $5 \%$ de cesáreas.

Una vez seleccionadas y ponderadas las variables de los índices, se creó una fórmula para cada uno de ellos. Tanto el ISSM como el ICI se definieron como la suma de las ponderaciones de las correspondientes variables (anexos 1 y 2 ). Con los datos de las variables de cada índice se hizo una planilla para cada una de las 112 provincias bolivianas. Aplicando las fórmulas a estos datos se obtiene un valor porcentual, es decir, el valor del índice respectivo.

A continuación se definieron tres categorías continuas para las puntuaciones obtenidas con cada índice, con el fin de facilitar su interpretación (cuadro 2). La categorización estuvo sujeta a consideraciones técnicas de los expertos y no a la simple división en tres porciones iguales de la puntuación total de 100. En el caso del ISSM, mientras más alto su valor, peor la situación materna, mientras que en el caso del ICI la interpretación es la opuesta: a mayor valor del índice, mejor capacidad instalada.

Por último, se definió la taxonomía de acuerdo con las posibilidades que otorgaba la combinación de ambos índices, que eran nueve (3 categorías del primer índice $x 3$ categorías del segundo) (cuadro 3).

\section{RESULTADOS}

De este modo se obtuvo un mapa nacional de la situación de salud materna y de la capacidad instalada en cada una de las 112 provincias bolivianas. La situación de salud de cada provincia aparece en una planilla con la puntua-

CUADRO 3. Agrupamiento de las 112 provincias bolivianas según la puntuación de los índices de la situación de salud materna (ISSM) y de la capacidad instalada (ICI). Bolivia, 2000

\begin{tabular}{|c|c|c|c|c|c|c|}
\hline \multirow[b]{2}{*}{ ISSM } & \multicolumn{6}{|c|}{$\mathrm{ICI}$} \\
\hline & \multicolumn{2}{|c|}{$\begin{array}{c}\text { Mala } \\
(<20 \text { puntos })\end{array}$} & \multicolumn{2}{|c|}{$\begin{array}{c}\text { Regular } \\
\text { (20 a } 40 \text { puntos) }\end{array}$} & \multicolumn{2}{|c|}{$\begin{array}{c}\text { Buena } \\
\text { (> } 40 \text { puntos) }\end{array}$} \\
\hline $\begin{array}{l}\text { Mala } \\
\qquad>45 \text { puntos })\end{array}$ & $A^{a}$ & $46^{b}$ & B & 11 & C & 5 \\
\hline Regular & & & & & & \\
\hline (25 a 45 puntos) & D & 4 & E & 10 & $\mathrm{~F}$ & 27 \\
\hline $\begin{array}{l}\text { Buena } \\
\qquad(<25 \text { puntos })\end{array}$ & G & 0 & $\mathrm{H}$ & 0 & 1 & 9 \\
\hline
\end{tabular}

a Las letras representan las categorías resultantes de la combinación de ambos índices.

${ }^{b}$ Las cifras representan el número de provincias en cada categoría.

ción de cada variable, el valor de cada índice $\mathrm{y}$, finalmente, su asignación taxonómica (primeras nueve letras del alfabeto), que resulta de la combinación de los dos índices. En el cuadro 2 se observa la distribución de las 112 provincias en cada una de las nueve casillas, de acuerdo con la taxonomía establecida para la salud materna.

Con estos datos se pudo seleccionar un número mínimo de provincias (9 con un total de 26 municipios y que implican a 17 distritos sanitarios) para intervención conjunta entre el Ministerio de Salud y Previsión Social y varios proyectos de la USAID y de otras agencias de cooperación. Esta selección se denominó "mínima", frente a otras dos con más provincias, denominadas "básica" (18 provincias) y "ampliada" (37 provincias). Para la selección mínima, las provincias debían reunir tres condiciones: a) más de 50000 habitantes, para efectos de impacto; b) 10 o más muertes maternas anuales estimadas, y c) regular o buena capacidad instalada. Con estos criterios, las provincias seleccionadas pertenecen a los grupos taxonómicos C y F.

\section{DISCUSIÓN}

Se presenta un método para identificar territorios críticos en términos de salud con propósitos de intervención, a partir de la utilización de información disponible. De este modo, el Ministerio de Salud y Previsión Social de Bolivia, las agencias de cooperación, las ONG, los municipios y otras instituciones afines e interesadas cuentan con un mapa de la situación global, en este caso de la salud materna, de las 112 provincias de los 9 departamentos bolivianos que permite identificar tres grupos de provincias para propósitos de intervención (selección mínima, básica y ampliada).

A partir de la premisa de que siempre hay más necesidades de salud que recursos para satisfacerlas (17), el procedimiento ha servido también para establecer alianzas entre instituciones de salud y civiles e incrementar la disponibilidad de recursos financieros mediante la elaboración de presupuestos compartidos. Además, se espera que también influya en otros actores que todavía no forman parte de la alianza, regulando y mejorando su participación. Esto permite reforzar la participación social, la capacidad administrativa de los sistemas de salud y la readecuación de los mecanismos de financiamiento (9).

Debido a que, más allá de la política y la tradición, existen razones de carácter administrativo y científico para establecer prioridades (17), este procedimiento puede aplicarse a otros problemas de salud, y también para establecer la situación de salud global. Para ello solo se necesita identificar variables que se registren habitualmente, asignarles una puntuación y elaborar la fórmula. Por último, después de las intervenciones es posible revisar la situación de cada variable $\mathrm{y}$, por lo tanto, de los índices respectivos, y compararlos con la situación basal establecida durante el primer mapeo. 


\section{REFERENCIAS}

1. Fondo de Población de las Naciones Unidas. Indicadores para evaluar la salud reproductiva y los programas pertinentes. Rev Panam Salud Publica 1998;3:62-68.

2. Organización Panamericana de la Salud. La administración estratégica. Lineamientos para su desarrollo: los contenidos educacionales. Washington, D.C.: OPS; 1994

3. Instituto Nacional de Estadística, Macro International Inc. Encuesta Nacional de Demografía y Salud, 1994. La Paz: INE; 1995.

4. World Health Organization, United Nations Children's Fund, United Nations Population Fund. Maternal mortality in 1995: estimates developed by WHO, UNICEF, UNFPA. Geneva: WHO/UNICEF/UNFPA; 2001

5. World Health Organization, United Nations Children's Fund. Revised 1990 estimates of maternal mortality: a new approach by WHO and UNICEF. Pan Am J Public Health 1997;1: $481-485$.

6. De la Galvez Murillo A, Pooley B. Mortalidad materna. En: Naciones Unidas. Remontando la pobreza. La Paz: EDOBOL; 2000. Pp. 145-165.

7. Organización Panamericana de la Salud. Evaluación del Plan de Acción Regional para la
Reducción de la Mortalidad Materna. Washington, D.C.: OPS; 1996.

8. Instituto Nacional de Estadística, Macro International Inc. Encuesta Nacional de Demografía y Salud, 1998. La Paz: INE; 1999.

9. Organización Panamericana de la Salud. Desarrollo y fortalecimiento de los sistemas locales de salud en la transformación de los sistemas nacionales de salud. Washington, D.C.: OPS; 1989.

10. Bolivia, Ministerio de Salud y Previsión Social, Proyecto Salud Materna y Neonatal, JHPIEGO. Redes de servicios sociales para el desarrollo de la salud materna y neonatal. La Paz: Ministerio de Salud y Previsión Social; 2000.

11. Sistema Nacional de Información en Salud. Atención del parto, ocupación camas de maternidad, médicos por 1000 habitantes, hospitales por 10000 habitantes, porcentaje de cesáreas. [Sitio en Internet] SNIS. Disponible en: http://www.sns.gov.bo/bolsns/apc.asp. Acceso el 30 marzo 2000.

12. Ministerio de Desarrollo Sostenible y Planificación, Unidad de Políticas de Población. Bolivia y sus municipios. Información poblacio- nal para la planificación, 1999. La Paz: UPP, 1999.

13. Programa de las Naciones Unidas para el Desarrollo. Desarrollo humano en Bolivia 1998. La Paz: Artes Gráficas Latina; 1998.

14. Maine D, Akalin MZ, Ward VM, Kamara A. The design and evaluation of maternal mortality. New York: Columbia University; 1997.

15. Inter-Agency Group for Safe Motherhood. The safe motherhood action agenda: priorities for the next decade. Colombo, Sri Lanka; Family Care International; 1997.

16. Médicos Consultores. Mortalidad materna en la ciudad de La Paz. La pérdida de una batalla por la vida. La Paz: Atenea SRL; 1994.

17. White F. De la evidencia al desempeño: cómo fijar prioridades y tomar buenas decisiones. Rev Panam Salud Publica. 1998;4:69-74.

Manuscrito recibido el 5 de diciembre de 2001 y aceptado para publicación, trás revisión, el 10 de junio de 2002.

\section{ANEXO 1. Fórmula para obtener el índice de la situación de la salud materna}

$$
\mathrm{SMx}=\left[\frac{(\mathrm{IDHa} \pm \mathrm{IDHx})}{\mathrm{IDHx}} \times 25\right]+\left(\frac{\mathrm{TMMx}}{\mathrm{TMMa}} \times 15\right)+\left(\frac{\mathrm{MMx}}{\mathrm{MMa}} \times 30\right)+[(1 \pm \mathrm{PSx}) \times 30]
$$

Donde:

SMx = Índice de la situación de la salud materna de la provincia $\mathrm{X}$

$\mathrm{IDHa}=$ Índice de desarrollo humano de la provincia de mayor desarrollo

$\mathrm{IDHx}$ = Índice de desarrollo humano de la provincia $\mathrm{X}$

$\mathrm{TMMx}=$ Tasa de mortalidad materna de la provincia $\mathrm{X}$ (prorrateada)

$\mathrm{TMMa}=$ Tasa de mortalidad materna de la provincia con la cifra más elevada

$\mathrm{MMx}=$ Número de muertes maternas esperadas en la provincia $\mathrm{X}$

$\mathrm{MMa}=$ Número de muertes maternas esperadas en la provincia con la cifra más elevada

$\mathrm{PSX}=$ Porcentaje de partos en servicios de obstetricia en la provincia $\mathrm{X}$

\section{ANEXO 2. Fórmula para obtener el Indice de Capacidad Instalada de Salud Materna}

$$
\mathrm{Clx}=\left(\frac{\mathrm{OCMx}}{\mathrm{OCMa}} \times 20\right)+\left(\frac{\mathrm{Mx}}{\mathrm{Ma}} \times 20\right)+\left(\frac{\mathrm{Hx}}{\mathrm{Ha}} \times 30\right)+(\mathrm{CCx})
$$

Donde:

$\begin{array}{ll}\mathrm{Clx} & =\text { Índice de Capacidad Instalada de la provincia } \mathrm{X} \\ \mathrm{OCMx} & =\text { Porcentaje de ocupación de camas de maternidad en la provincia } \mathrm{X} \\ \mathrm{OCMa} & =\text { Porcentaje más alto de ocupación de camas de maternidad } \\ \mathrm{Mx} & =\text { Número de médicos por } 10000 \text { habitantes en la provincia } \mathrm{X} \\ \mathrm{Ma} & =\text { Número más elevado de médicos por } 10000 \text { habitantes } \\ \mathrm{Hx} & =\text { Número de hospitales por } 10000 \text { habitantes en la provincia } \mathrm{X} \\ \mathrm{Ha} & =\text { Número más elevado de hospitales por } 10000 \text { habitantes } \\ \mathrm{CC} & =\text { Capacidad para realizar cesáreas (en una escala de } 0 \text { a } 30)\end{array}$


ABSTRACT Objective. In early 2000 the Ministry of Health and Social Welfare of Bolivia brought together a group of experts who, using available information, developed a method that made it possible to identify critical maternal and neonatal health sections of the

\section{Using indicators to identify regions with critical maternal health conditions}

country and to create a map of the health situation and of the existing health-services capacity in the 112 provinces of Bolivia. The objective of this piece is to describe the method that those experts created and applied.

Methods. Two indices were created, one for the health situation and the other for the existing health-services capacity. The steps followed in this process were: 1) identifying the variables included in each index, 2) weighting the variables in each index, 3) creating a mathematical formula for each index, 4) preparing a list with the data from each province for the chosen variables and with the percentage for each province for each index, obtained by using the respective formula, 5) setting three continuous-data categories for each index, and 6) defining the taxonomy that was possible by combining the results of the two indices.

Results. Applying this approach, a national map of the maternal health situation and of the existing capacity in each of the 112 Bolivian provinces was developed. This made it possible to choose a small number of provinces where the Ministry of Health and Social Welfare could work with other institutions to carry out joint interventions. The 9 selected provinces have a total of 26 municipalities, which include 17 health districts and which have $29 \%$ of the population of the country, $33 \%$ of the maternal deaths, and an estimated $35 \%$ of the early neonatal deaths.

Conclusions. Using available information, this method generated a map of the overall maternal health situation in the 112 provinces of Bolivia and made it possible to identify critical geographical areas for health interventions.

\title{
Programas de Magister en Salud Pública y Bioestadística, 2003
}

La Escuela de Salud Pública de la Facultad de Medicina de la Universidad de Chile en Santiago ofrece desde 1981 los Programas de Magister en Salud Pública y en Bioestadística, dirigidos a profesionales de las áreas de salud y políticas públicas de Chile y América Latina.

El Magister en Salud Pública tiene por objetivo proporcionar una sólida formación académica, orientada hacia la medicina social, que prepare a los graduados para su desempeño en las áreas de docencia, investigación y administración. El estudiante podrá elegir entre las siguientes áreas de profundización: Economía de la Salud, Epidemiología, Políticas y Gestión en Salud, Promoción de la Salud, Salud Ocupacional, Salud y Ambiente.

El Magister en Bioestadística se encamina a formar profesionales de alto calibre en la aplicación de métodos cuantitativos en el campo de la salud pública. El título habilita a quien lo posee para la docencia, la investigación y el asesoramiento de instituciones e investigadores dedicados a esta disciplina.

Los programas tienen una duración de un año lectivo, al cabo del cual se redacta una tesis. La fecha límite para el recibo de las solicitudes es el 22 de noviembre de 2002.

\author{
Información: \\ Universidad de Chile \\ Facultad de Medicina \\ Coordinación de Posgrado \\ Independencia 939 \\ Santiago, Chile \\ Teléfono: (56-2) 7686150
}

Fax: (56-2) 7355582

Correo electrónico: jcumsill@machi.med.uchile.cl

Internet: http://www.saludpublica.uchile.cl 